This item was submitted to Loughborough's Research Repository by the author.

Items in Figshare are protected by copyright, with all rights reserved, unless otherwise indicated.

\title{
Evaluating feedback time delay during perturbed and unperturbed balance in handstand
}

PLEASE CITE THE PUBLISHED VERSION

http://dx.doi.org/10.1016/j.humov.2016.04.011

PUBLISHER

(C) Elsevier

VERSION

AM (Accepted Manuscript)

PUBLISHER STATEMENT

This paper was accepted for publication in the journal Human Movement Science and the definitive published version is available at https://doi.org/10.1016/j.humov.2016.04.011

LICENCE

CC BY-NC-ND 4.0

\section{REPOSITORY RECORD}

Blenkinsop, Glen, Matthew Pain, and Michael Hiley. 2016. "Evaluating Feedback Time Delay During Perturbed and Unperturbed Balance in Handstand”. Loughborough University. https://hdl.handle.net/2134/21457. 


\title{
Evaluating feedback time delay during perturbed and unperturbed balance in handstand
}

\author{
Glen M Blenkinsop, Matthew TG Pain, and Michael J. Hiley \\ School of Sport, Exercise and Health Sciences, Loughborough University, Leicestershire, LE11 3TU, UK
}

\begin{abstract}
Feedback delays in balance are often assessed using muscle activity onset latencies in response to discrete perturbations. The purpose of the study was to calculate EMG latencies in perturbed handstand, and determine if delays are different to unperturbed handstand. Twelve national level gymnasts completed 12 perturbed and 10 unperturbed (five eyes open and five closed) handstands. Forearm EMG latencies during perturbed handstands were assessed against delay estimates calculated via: cross correlations of wrist torque and COM displacement, a proportional and derivative model of wrist torque and COM displacement and velocity (PD model), and a PD model incorporating a passive stiffness component (PS-PD model). Delays from the PD model (161 $\pm 14 \mathrm{~ms}$ ) and PSPD model (188 $\pm 14 \mathrm{~ms}$ ) were in agreement with EMG latencies (165 $\pm 14 \mathrm{~ms})$. Cross correlations of COM displacement and wrist torque provided unrealistically low estimates (5 $\pm 9 \mathrm{~ms}$ ). Delays were significantly lower during perturbed (188 $\pm 14 \mathrm{~ms}$ ) compared to unperturbed handstand (eyes open: $207 \pm 12 \mathrm{~ms}$; eyes closed: $220 \pm 19 \mathrm{~ms}$ ). Significant differences in delays and model parameters between perturbed and unperturbed handstand support the view that balance measures in perturbed testing should not be generalised to unperturbed balance.
\end{abstract}

Keywords: handstand balance, EMG latencies, cross correlations, regression modelling

\section{INTRODUCTION}

The control of posture involves a feedback system which processes visual, vestibular, and somatosensory inputs and executes neuromuscular actions to maintain equilibrium (Winter, 1995; Wade and Jones, 1997; Horak, 2006). Feedback time delays complicate control tasks, placing constraints on the strategies employed and limiting the stability of the system (Bottaro et al., 2005; Peterka, 2000; Suzuki et al., 2012; Vette et al., 2010). Likewise, the mechanical stability of the system places constraints on the strategies employed and limits the range of delays possible for stable control to be established (Suzuki et al., 2012). Control models of human balance must include the appropriate level of mechanical instability and a realistic time delay to accurately simulate postural dynamics. Models that incorporate large amounts of noise to replicate human sway typically use delays of 80-100 ms (van der Kooij et al., 2005; Vette et al., 2010), whereas models using intermittent control to replicate human sway typically use delays of $180-200 \mathrm{~ms}$ (Bottaro et al., 2008; Suzuki et al., 2012). The different conclusions drawn from these models may be due to the different feedback time delays used.

There are several possible sources of instability in human posture. The majority of previous literature has focused on the examination of postural control during upright stance, however, this is not the only form of human balance (Slobounov and Newell, 1996). Balancing in handstand is mechanically less stable than balancing in normal upright stance, with a reduced base of support, a higher centre of mass, and a reduced maximal strength in the controlling joints. Regardless, from a control perspective, the two tasks are equivalent, requiring estimation of the spatial arrangement and motion of the body, and implementing a multi-joint strategy to preserve orientation and configuration within certain constraints (Yeadon and Trewartha, 2003). Handstand balance performed by experienced gymnasts provides 
an alternative perspective to normal upright stance for understanding this complex system.

During quiet stance an ankle strategy is dominant, where the motion of the centre of mass (COM) is controlled by torque about the ankle joint with synergistic torques about superior joints to maintain a fixed body configuration (Nashner and McCollum, 1985; Runge et al., 1999). The ankle strategy is analogous to a wrist strategy while balancing in handstand, where the system can be modelled as a single segment inverted pendulum controlled by wrist joint torque (Yeadon and Trewartha, 2003). The feedback time delay from an initial perturbing motion to muscle force production comprises sensory delay, neurological delay, and electromechanical delay (Figure 1). Sensory delay arises as it takes time for receptors to reach a sensory threshold; it is expected to be related to speed of motion and sensory acuity. Neurological delay represents the time for sensory transduction, neural processing, and motor signal transmission (Peterka, 2002), and is expected to be relatively constant within an individual. Electromechanical delay is the time from muscle activation until the onset of muscle force production, which is approximately 13 to 55 ms (Cavanagh and Komi, 1979; Tillin et al., 2010; Zhou et al., 1995). This wide variation in electromechanical delay can be due to differences in musculotendon tension and slack length (Muraoka et al., 2004).

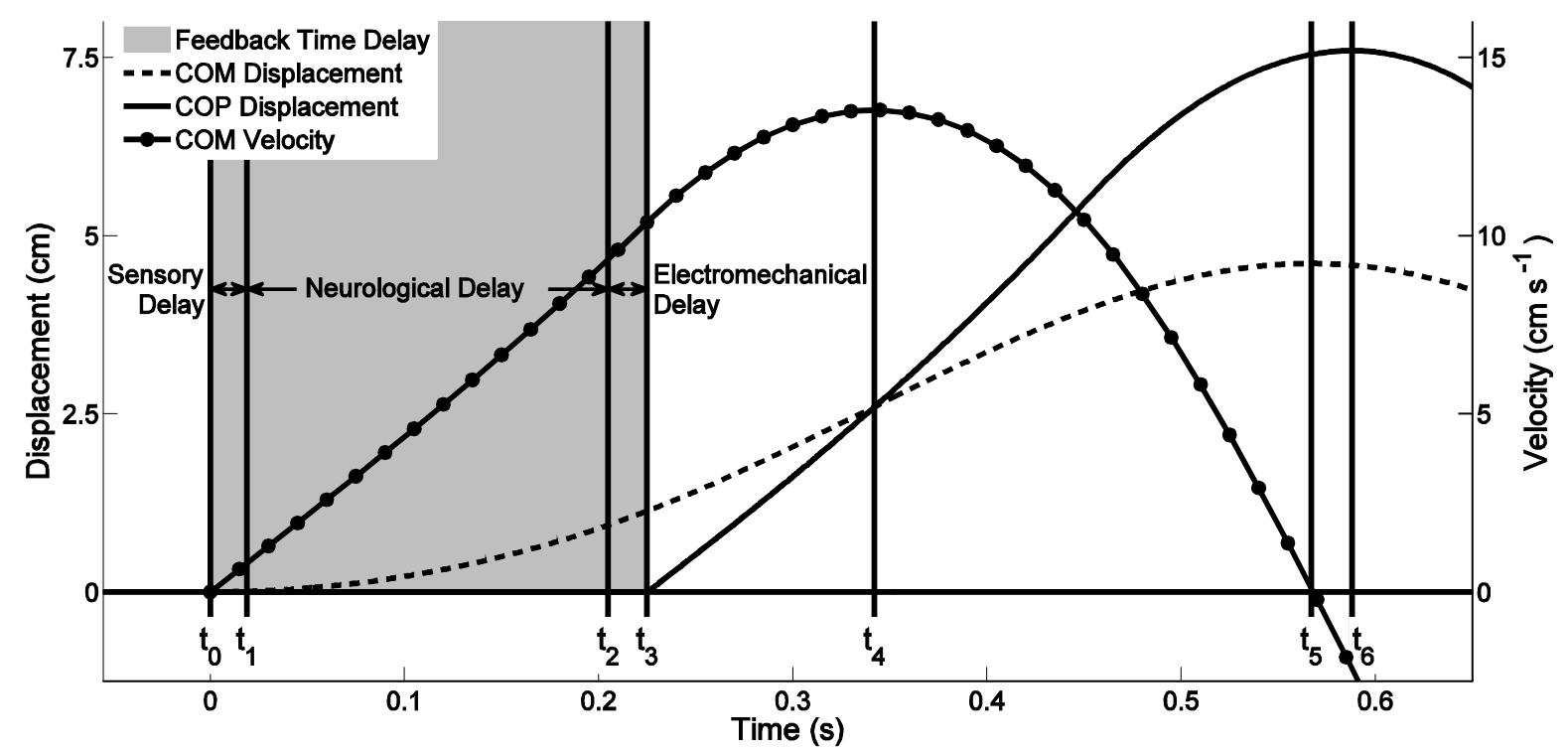

Figure 1. A theoretical description of COP and COM motion of a single inverted pendulum model of balance controlled by a reactive strategy $\left[\mathrm{t}_{0}=\right.$ initial motion; $\mathrm{t}_{1}=$ sensory threshold is reached and motion is detected; $t_{2}=$ muscle activated; $t_{3}=$ muscle force is produced; $t_{4}=$ COP crosses COM, joint torque is higher than torque due to gravity, and peak COM velocity is reached; $t_{5}=$ peak COM displacement; $t_{6}=$ peak COP displacement $]$

The time from a discrete platform perturbation until the onset of muscle activation, detected via electromyography (EMG), will describe the time from the initiation of movement until muscle activation $\left(\mathrm{t}_{2}-\mathrm{t}_{0}\right)$. In perturbed standing EMG latencies are approximately 65 to $130 \mathrm{~ms}$, with corresponding changes in joint torques occurring around 30 ms later (Horak et al., 1989; Nashner et al., 1979), representing the electromechanical delay $\left(t_{3}-t_{2}\right)$. However, these values may not be typical of unperturbed balance as static and dynamic posturography techniques address different aspects of the postural control system (Baratto et al., 2002).

Feedback time delay estimates in quiet stance have been determined from cross correlations of centre of mass (COM) and centre of pressure (COP) 
trajectories, which describe the time from peak COP displacement to peak COM displacement $\left(\mathrm{t}_{6}-\mathrm{t}_{5}\right)$ or peak COM velocity $\left(\mathrm{t}_{6}-\mathrm{t}_{4}\right)$. The delay between COP and COM displacements is normally zero and has been suggested as evidence of a passive control system (Winter et al., 1998, 2003). However, it has also been suggested this is evidence of an active anticipatory feedforward control process (Gatev et al., 1999). These studies assume that the postural control strategy relies primarily on segment or whole body displacement information from sensory inputs, however, the postural control system adopts a strategy that relies heavily on velocity information during quiet stance (Kiemel et al., 2002; Masani et al., 2003). It has also been shown that sensory thresholds for displacement at the ankle (Clark et al., 1985; Fitzpatrick and McCloskey, 1994) and metacarpophalangeal joint (Clark et al., 1985) are reduced when the joint is moved more quickly. Assessment of feedback time delay during unperturbed balance may require consideration of displacement and velocity of the body together, as utilised in a proportional and derivative (PD) control model of balance.

The displacement and velocity of the COM are used in a PD control model of balance to determine the required joint torques to control COM motion (Masani et al., 2003, 2006; Morasso et al., 1999; Bottaro et al., 2005, 2008). Yeadon and Trewartha (2003) applied this principle to experimental data in handstand balance, using delayed regressions of wrist joint torque against COM displacement and velocity to provide estimates of feedback time delay of 160 to $240 \mathrm{~ms}$. The assumption for delayed regression models is that the total joint torque is generated actively based on feedback from position and velocity sensors. Ankle joint torque during standing balance has been modelled as a composite of: active torque (12\%), passive stiffness $(19 \%)$, and a bias torque (69\%) required to maintain the average position (Jacono et al., 2004). However, any torque caused by passive stiffness will have approximately zero delay, resulting in underestimates of feedback time delays if not considered separately. To date no study has evaluated the contribution of passive stiffness to maintaining balance in handstand.

Feedback time delay estimates from both cross correlations in unperturbed upright stance and delayed regression models in unperturbed handstand balance fall outside of estimated delays (EMG latency plus electromechanical delay) previously found in perturbed standing. Although feedback time delays calculated during dynamic posturography tests should not be assumed to apply to unperturbed balance, it remains unclear if these differences are due to changes in the task being evaluated or limitations in the analysis methods employed. The aim of the present study was to evaluate cross correlation and delayed regression models against EMG latencies from discrete perturbations in handstand balance with regard to feedback time delays, and to determine if these delays are different between perturbed and unperturbed handstand balance. It is hypothesised that including passive stiffness in the delayed regression models will provide a better estimate of feedback time delay which will be significantly lower during perturbed compared to unperturbed handstand balance.

\section{METHODS}

\section{Subjects}

Twelve national level gymnasts experienced at balancing in handstand were recruited for the study, including nine males (age: $23.1 \pm 3.6$ [mean \pm SD] years; mass: $69.9 \pm 2.2 \mathrm{Kg}$; height: $1.73 \pm 0.05 \mathrm{~m}$ ) and three females (age: $20.5 \pm 0.7$ years; mass: $57.9 \pm 1.9 \mathrm{Kg}$; height: $1.64 \pm 0.02 \mathrm{~m}$ ). All gymnasts were able to balance in handstand for at least 30 seconds whilst maintaining a static base of support. 
Gymnasts were free from injury during the testing period and gave written informed consent for participation in the study that was approved by the University Ethical Advisory Committee.

\section{Procedure}

During all trials subjects were instructed to maintain a static base of support, and attempt to remain in, or return to, the standard handstand position of fully extended arms, trunk, and legs with feet together. A change to the base of support, such as a shuffle or a free limb contacting the ground was considered a failure to maintain balance, and the trial was halted. Perturbed and unperturbed balance was assessed in two separate sessions approximately one week apart. Gymnasts completed unperturbed balance trials for a maximum of 30 seconds duration in handstand with eyes open and eyes closed conditions. Each condition was completed in a block of five trials with a minimum 60 seconds rest between each trial and 20 minutes rest between blocks.

Perturbed balance with eyes open was assessed with 12 randomised discrete platform translations, with three trials for each of four types of perturbation: forwards small, forwards large, backwards small, and backwards large. Small perturbations had an amplitude of $0.05 \mathrm{~m}$, peak velocity of $\pm 0.1 \mathrm{~m} \mathrm{~s}^{-1}$, and peak acceleration of \pm $0.6 \mathrm{~m} \mathrm{~s}^{-2}$ whereas large perturbations had an amplitude of $0.10 \mathrm{~m}$, peak velocity of \pm $0.2 \mathrm{~m} \mathrm{~s}^{-1}$, and peak acceleration of $\pm 1.2 \mathrm{~m} \mathrm{~s}^{-2}$. Gymnasts were required to attain a stable balanced handstand position before data collection began, after which 1 to 3 seconds of static balance was performed before the initiation of the perturbation. Perturbed trials were stopped when either the subject failed to maintain balance with a static base of support or when the experimenter judged the subject had returned to a stable balanced position.

\section{Data Collection}

All balance trials were completed on a CAREN system (Motek Medical), with perturbations created by the translation of a six degree of freedom Stewart platform controlled by a custom script written in the Motek Medical D-Flow software. Kinematic data were collected using nine T20 Vicon (Vicon, Oxford Metrics Group) cameras operating at $200 \mathrm{~Hz}$. Fifty three spherical markers of $14 \mathrm{~mm}$ diameter were used to divide the body into 18 segments. Individual segmental inertial parameters were obtained from anthropometric measurements using the inertia model of Yeadon (1990). Kinetic data were collected via two $0.4 \mathrm{~m} \times 0.6 \mathrm{~m}$ force plates (Bertec FP4060-07) embedded in the Stewart platform with a sample frequency of $2000 \mathrm{~Hz}$. In order to correct for COP and inertial force errors introduced into the force plate measures (Preuss and Fung, 2004) four additional markers were placed outside the area of the force plates to track the motion of the platform.

Surface EMG (Delsys Trigno ${ }^{\mathrm{TM}}$ wireless EMG system, Delsys Inc.) was digitally recorded from the right and left wrist flexor and extensor muscles at $2000 \mathrm{~Hz}$ following SENIAM guidelines. Each EMG signal was amplified ( $\times 1000$ into $\pm 5 \mathrm{v}$ ) then underwent digital to analogue conversion. The digital to analogue conversion introduced a $48 \mathrm{~ms}$ offset into the EMG signal output (Trigno ${ }^{\text {TM }}$ Wireless System User's Guide, Delsys Inc.) which was checked and accounted for before synchronisation with other data. All analogue data were passed through the same analogue to digital converter within the Vicon Giganet unit to synchronise force, EMG, and kinematic data. 


\section{Data Processing}

The raw EMG data were filtered with a fourth order, zero lag, band-pass Butterworth filter with cut-off frequencies of 20 and $450 \mathrm{~Hz}$ (De Luca et al., 2010), before being rectified and scaled to EMG from maximal voluntary contractions (MVC), obtained from independent maximal isometric trials. Force, moment, and COP data were down sampled to $200 \mathrm{~Hz}$ and combined with kinematic data for inverse dynamics calculations. Low pass cut-off frequency estimates for force, COP displacements, and marker displacements were 4 to $9 \mathrm{~Hz}$ based on residual analysis and 6 to $10 \mathrm{~Hz}$ based on power spectral analysis. To provide consistency across trials, and to reduce the possibility of attenuating the true signal by over filtering, all force, COP and marker data were filtered using a fourth order, zero lag, low-pass Butterworth filter with a cut-off frequency of $10 \mathrm{~Hz}$.

Quaternion algebra (Dumas et al., 2004) was used to calculate segment COM linear displacements and accelerations, and segment angular velocities and accelerations. Kinematic, inertia, and kinetic data were combined to calculate threedimensional joint forces and moments using wrench notation (Dumas et al., 2004). Right and left wrist joint moments were summed to provide a total wrist moment about a global mediolateral axis.

\section{Data Analysis}

Visual inspection was used to determine the initiation of platform perturbations from raw force data, and rectified EMG onset (Hodges and Bui, 1996; Moretti et al., 2003; Pulkovski et al., 2008; Tillin et al., 2013) (Figure 2a). Large amounts of EMG activity were present throughout the duration of handstand trials, making it extremely difficult to determine the onset of a single muscular response to platform movements. Consequently, analysis was only performed on those trials where a clear response to the platform perturbation was evident, determined by a period of low EMG activity (less than $10 \%$ of peak) at least $50 \mathrm{~ms}$ before and after the initiation of the perturbation. No fewer than seven trials were analysed for any one gymnast.

Estimates of feedback time delays were calculated by employing cross correlations (Gatev et al., 1999; Masani et al., 2003, 2006; Winter et al., 1996, 1998) and delayed regression models (Yeadon and Trewartha, 2003). Cross correlations to determine time delays between: anteroposterior COM displacement and wrist joint torque (Figure 2b), anteroposterior COM displacement and EMG, and wrist joint torque and EMG, were implemented in Matlab. Yeadon and Trewartha's PD model (2003) was used to determine the delay between anteroposterior COM motion and the corresponding wrist joint torque (Eq. 1 and Figure 2c). To assess the contribution of a passive stiffness mechanism in handstand a further regression model (PS-PD model) was introduced with a passive stiffness parameter based on COM displacement with zero delay (Eq. 2):

$$
\begin{gathered}
T_{(t)}=p_{2} x_{\left(t-t_{d}\right)}+d \dot{x}_{\left(t-t_{d}\right)} \\
T_{(t)}=p_{1} x_{(t)}+p_{2} x_{\left(t-t_{d}\right)}+d \dot{x}_{\left(t-t_{d}\right)}
\end{gathered}
$$

where wrist joint torque $T$ at time $t$ is based on the displacement $x$ at time $t$ and the displacement $x$ and velocity $\dot{x}$ at an earlier time due to the feedback time delay $t_{d}$. Proportional gains $p_{1}$ and $p_{2}$ and the derivative gain $d$ were determined via delayed regressions of wrist joint torque against COM displacement $\left(p_{2}\right)$ and velocity $(d)$ at earlier times and COM displacement with no delay $\left(p_{1}\right)$. Regressions were repeated 
with delays from -500 to $500 \mathrm{~ms}$ in $5 \mathrm{~ms}$ intervals. The time delay $t_{d}$ was chosen as the delay that produced the largest $\mathrm{R}^{2}$ value from the regressions. Regression parameters were then used with COM motion to recreate the predicted signal. The percentage contributions of wrist joint torque from the three proportional and derivative gains were then calculated, indicating the relative importance of sensory feedback and passive stiffness in controlling handstand balance.
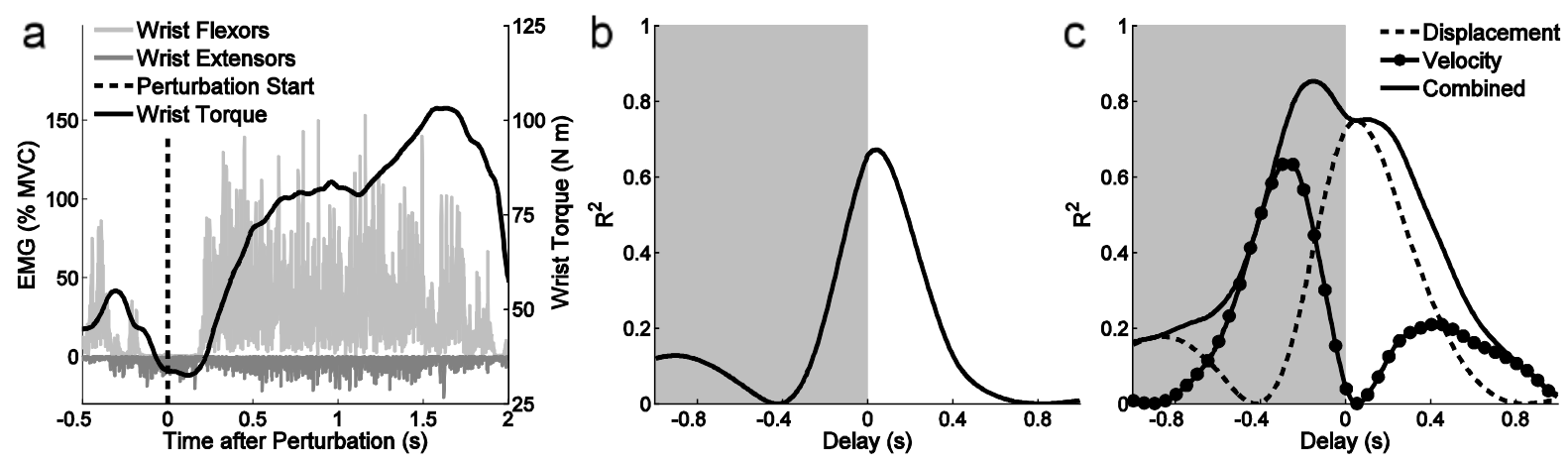

Figure 2. Examples of the three methods for determining feedback time delay, including: a) the EMG and wrist torque response to a large forwards platform perturbation; b) cross correlation of COM displacement and wrist joint torque, with a positive delay (white area) indicating COM peaks after torque; and c) delayed regression of COM displacement and velocity against wrist joint torque, with a negative delay (grey area) indicating COM motion peaks before torque.

\section{Statistical Analysis}

Trials were combined to create a mean score for each subject under each condition (perturbed, unperturbed with eyes open, and unperturbed with eyes closed) before further analysis. Repeated measures ANOVA's were used to assess the differences between perturbed and unperturbed handstand with eyes open and eyes closed conditions for: estimated feedback time delay, and the contribution of total wrist joint torque from $p_{1}, p_{2}$, and $d$. Post hoc tests were made using multiple repeated measures $t$-tests with a Bonferroni correction. All data were assessed for normality and sphericity by the one-sample Kolmogorov-Smirnov test and Mauchly's test of sphericity. A Greenhouse-Geisser correction was used for any data that was found to violate the assumption of sphericity. The significance level was set at 0.05 .

\section{RESULTS}

Estimates of feedback time delay in perturbed handstands were $165 \pm 14 \mathrm{~ms}$ based on EMG latency, $161 \pm 14 \mathrm{~ms}$ based on the PD model, $188 \pm 14 \mathrm{~ms}$ based on the PS-PD model, and $5 \pm 9 \mathrm{~ms}$ based on cross correlations of COM displacements to wrist joint torque (Table 1). Additional cross correlations showed COM displacements lagged behind forearm EMG by $83 \pm 26 \mathrm{~ms}$ (range: 36 to $138 \mathrm{~ms}$ ), and wrist joint torques lagged behind forearm EMG by $90 \pm 10 \mathrm{~ms}$ (range: 74 to 101 $\mathrm{ms})$.

There was a significant difference between perturbed handstands and unperturbed handstands for: feedback time delay, torque from p_1, torque from p_2, and torque from $d$ (Table 2). Further comparisons revealed these significant differences were between perturbed handstands and unperturbed handstands with eyes open, and perturbed handstands and unperturbed handstands with eyes closed for all variables. An additional significant difference was also found between 
unperturbed handstands with eyes open and unperturbed handstands with eyes closed for feedback time delay (Table 2 and Figure 3).

Table 1. EMG latencies, and feedback time delay estimates from delayed regression models (PD and PS-PD) and cross correlation (XC) during perturbed trials.

\begin{tabular}{ccccccccccc}
\hline & \multirow{2}{*}{$\begin{array}{c}\text { EMG } \\
\text { Latency }\end{array}$} & \multicolumn{3}{c}{ PD Model } & \multicolumn{3}{c}{ PS-PD Model } & \multicolumn{3}{c}{ XC: Torque - COM } \\
\cline { 2 - 11 } & Delay & $\mathrm{R}^{2}$ & diff & Delay & $\mathrm{R}^{2}$ & diff & Delay & $\mathrm{R}^{2}$ & diff \\
\hline Mean & 165 & 161 & 0.75 & -3 & 188 & 0.76 & 23 & 5 & 0.77 & -160 \\
SD & 14 & 14 & 0.06 & 20 & 14 & 0.06 & 13 & 9 & 0.06 & 20 \\
Max & 192 & 194 & 0.83 & 29 & 207 & 0.83 & 42 & 16 & 0.84 & -123 \\
Min & 137 & 142 & 0.67 & -50 & 162 & 0.67 & 3 & -9 & 0.64 & -186 \\
\hline
\end{tabular}

Note: All EMG latencies and feedback time delays are presented in milliseconds; diff = difference to EMG latencies (mean, standard deviation, minimum, and maximum of estimate minus EMG latency)

Table 2. Feedback time delay estimates from the PS-PD method, and the percentage of wrist torque from the three coefficients for perturbed $(P)$ and unperturbed handstands with eyes open (EO) and eyes closed $(\mathrm{EC})$.

\begin{tabular}{|c|c|c|c|c|c|}
\hline & Delay (ms) & $\mathrm{R}^{2}$ & p1 (\%) & p2 (\%) & $d(\%)$ \\
\hline & Mean \pm SD & Mean \pm SD & Mean \pm SD & Mean \pm SD & Mean \pm SD \\
\hline Perturbed & $188 \pm 14$ & $0.76 \pm 0.06$ & $25.5 \pm 11.5$ & $50.8 \pm 13.3$ & $23.6 \pm 3.8$ \\
\hline Eyes Open & $207 \pm 12$ & $0.78 \pm 0.03$ & $5.0 \pm 1.3$ & $65.4 \pm 2.8$ & $29.6 \pm 2.6$ \\
\hline Eyes Closed & $220 \pm 19$ & $0.79 \pm 0.03$ & $6.3 \pm 5.2$ & $64.3 \pm 6.6$ & $29.4 \pm 3.0$ \\
\hline \multicolumn{6}{|l|}{ ANOVA: } \\
\hline F statistic & 21.2 & 1.9 & 27.4 & 10.9 & 14.46 \\
\hline$p$ value & $<0.001$ & $>0.05$ & $<0.001$ & $<0.001$ & $<0.001$ \\
\hline \multicolumn{6}{|l|}{ T-Test: } \\
\hline P vs. EO & $-4.3^{\star \star}$ & - & $5.7^{\star \star \star}$ & $-3.5^{\star}$ & $-4.0^{\star \star}$ \\
\hline P vs. EC & 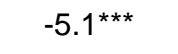 & - & $5.4^{\star \star \star}$ & $-3.5^{\star}$ & $-4.0^{\star \star *}$ \\
\hline EO vs. EC & $-3.3^{*}$ & - & -0.7 & 0.5 & 0.3 \\
\hline
\end{tabular}

Note: coefficients represent the percentage of torque estimated by: a passive stiffness mechanism $\left(p_{1}\right)$, delayed COM displacement $\left(p_{2}\right)$, and delayed COM velocity $(d)$. Significant t-tests are indicated by * $(p<0.01)$, ** $(p<$ $0.005)$, and ${ }^{* \star *}(p<0.001)$

\section{DISCUSSION}

Signal delays are an important feature of any biological system, with large delays reducing stability and complicating the control. Handstand balance performed by experienced gymnasts provides an alternative perspective to normal upright stance for understanding this complex system. The purpose of the present study was to evaluate feedback time delay in perturbed and unperturbed balance in the challenging task of handstand. During platform perturbations delayed regression models provided estimates of feedback time delay that were in agreement with EMG latencies, whereas cross correlations of COM displacement and wrist joint torque provided estimates that were unrealistically low (Table 1). These results support the hypothesis that delayed regression models are an appropriate tool for determining feedback time delay during handstand balance. Feedback time delays were significantly shorter during perturbed handstand than unperturbed handstand, supporting the hypothesis that feedback time delays are lower during perturbed testing than during static balance. 

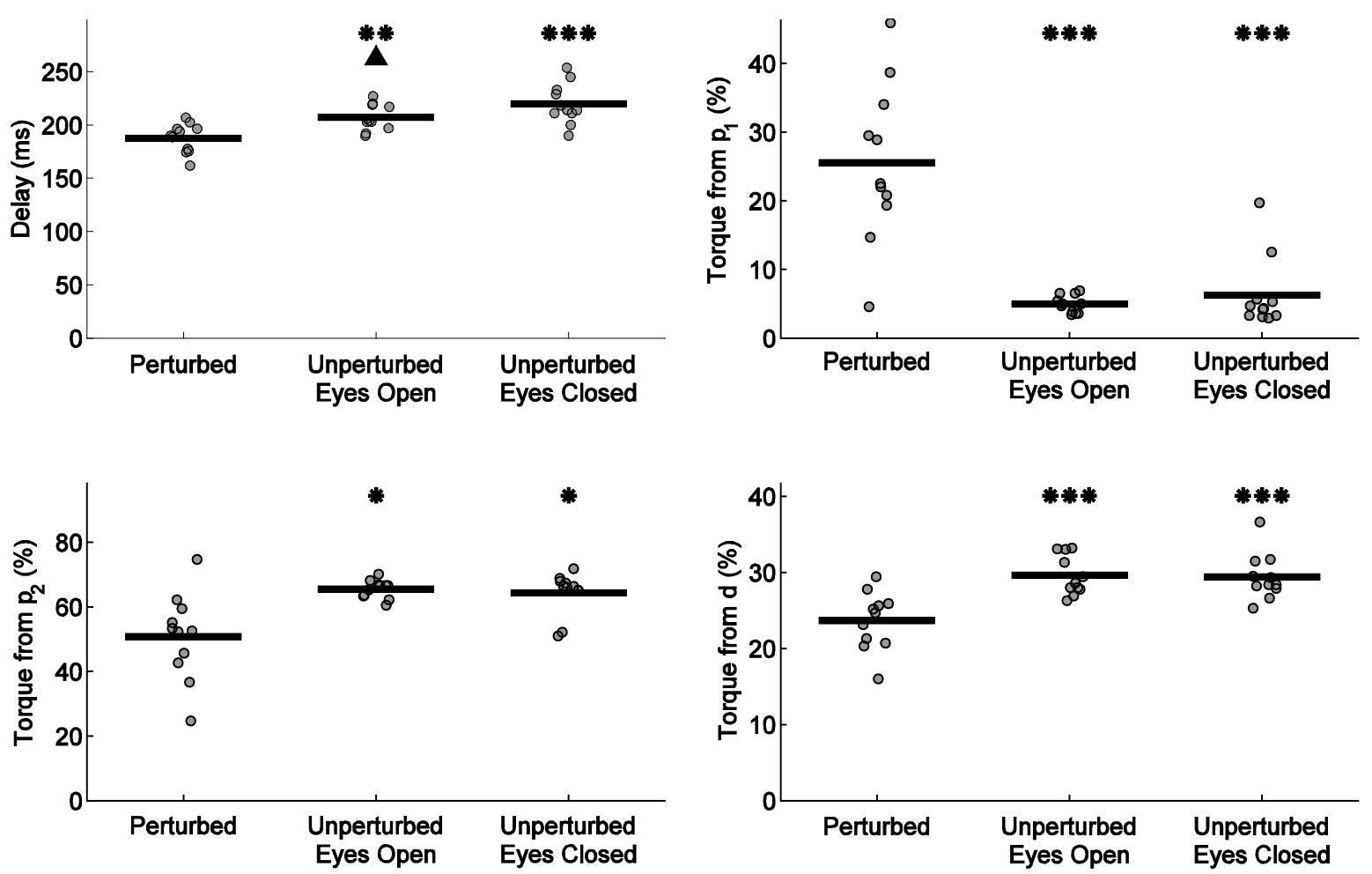

Figure 3. Mean feedback time delay estimates from the PS-PD method, and the percentage of wrist torque from the three coefficients for perturbed and unperturbed handstand. [a significant difference between unperturbed handstand with eyes open and eyes closed is indicated by $\Delta(p<0.01)$; a significant difference to perturbed handstand is indicated by $*(p<0.01)$, $* *(p<0.005)$ and **** $(p<0.001)]$

In order to apply delayed regression models, the assumption that a PD controller is used to stabilise a single segment inverted pendulum is required (derivation in Yeadon and Trewartha, 2003). If these assumptions are realistic the delayed regression will give the proportional and derivative gains used in the PD controller at a time delay that matches the feedback time delay of the system. Using the delayed regression models in the present study is a simplification of handstand balance and thus small violations are expected. The dominant control strategy in standing is an ankle strategy, which is maintained during small perturbations. However, during larger perturbations individuals begin to employ a hip strategy to help maintain balance (Nashner and McCollum, 1985; Runge et al., 1999). Although the dominant control strategy in handstand is a wrist strategy, several other control strategies can also be employed to help maintain balance, such as an elbow, shoulder, or hip strategy (Kerwin and Trewartha, 2001; Slobounov and Newell, 1996; Yeadon and Trewartha, 2003). As balance in handstand becomes more precarious it is likely that these other control strategies may be employed, violating the assumption of a single segment inverted pendulum. Furthermore, using a wrist strategy to control handstand balance requires synergistic torques about superior joints to maintain a fixed body configuration (Yeadon and Trewartha, 2003). Errors in sensory inputs or motor outputs at these joints will result in unwanted movement away from a fixed configuration, violating the assumption of a single segment inverted pendulum. Reasonable $\mathrm{R}^{2}$ values of 0.67 to 0.83 for perturbed handstand and 0.73 to 0.83 for unperturbed handstand (Table 2) indicates these violations are minimal and it is appropriate to model handstand balance as a single segment inverted pendulum with a PS-PD control model. Similar $\mathrm{R}^{2}$ values suggest that a wrist strategy is the dominant control strategy in both perturbed and unperturbed balance. 
Future studies may wish to explore this further by analysing a larger range of perturbation magnitudes than was employed in the present study.

The PS-PD delayed regression model provided estimates that were 3 to $42 \mathrm{~ms}$ longer than EMG latencies, whereas the PD model estimates differed by -50 to 29 ms (Table 1). The two regression models will provide an estimate of the time from the initiation of the perturbation until muscle force production $\left(t_{3}-t_{0}\right)$ (Figure 1). EMG latencies will determine the time from the initiation of the perturbation until muscle activation $\left(\mathrm{t}_{2}-\mathrm{t}_{0}\right)$ (Figure 1$)$. Therefore, the difference between EMG latencies and feedback time delay from the regression models will give an estimate of the electromechanical delay from muscle activation until force production $\left(t_{3}-t_{2}\right)$ (Figure 1). Only the PS-PD model delays (3 to $42 \mathrm{~ms}$ ) are in the correct region to account for an electromechanical delay of 13 to 55 ms (Cavanagh and Komi, 1979; Muraoka et al., 2004; Tillin et al., 2010; Zhou et al., 1995). As expected, feedback time delays from the PD model are somewhat underestimated, as torques caused by passive stiffness are not explicitly considered in the model. Feedback time delay estimates from cross correlations of wrist joint torque and COM displacement were 123 to 186 ms shorter than EMG latencies during perturbed handstands. Additional cross correlations showed that COM displacements lagged behind forearm EMG by 36 to $138 \mathrm{~ms}$, and wrist joint torques lagged behind forearm EMG by 74 to $101 \mathrm{~ms}$. It would appear that from these results that cross correlations are an inappropriate tool for determining feedback time delay in human balance (Morasso and Schieppati, 1999; van der Kooij et al., 2005).

During perturbed handstand balance the platform motion results in a rapid stretch of the musculotendinous unit at the wrist. Torque from a passive stiffness mechanism will have zero delay, resulting in an underestimation of feedback time delays if not included. The Yeadon and Trewartha PD model (2003) did not have a passive stiffness mechanism, however, the authors filtered out joint torques and COM motion above $2 \mathrm{~Hz}$ to remove high frequency vibrations due to muscle stiffness. This resulted in delays that were approximately $40 \mathrm{~ms}$ longer than those obtained without filtering. The PD model in the present study did not perform this type of filtering on calculated joint torques, however, the PS-PD model addressed the issue by including an extra term with zero delay. During unperturbed handstands, feedback time delays of 190 to $227 \mathrm{~ms}$ (eyes open) and 190 to $254 \mathrm{~ms}$ (eyes closed) obtained from the PS-PD model are similar to the 160 to $240 \mathrm{~ms}$ found by Yeadon and Trewartha (2003). Interestingly, Yeadon and Trewartha (2003) also subtracted $40 \mathrm{~ms}$ from feedback time delays as an approximation of the extra time in the calculation for joint torque to rise to the required level. In the present study, comparisons of feedback time delay and EMG latency in perturbed handstand would suggest an electromechanical delay of $23 \pm 13 \mathrm{~ms}$, and comparisons of feedback time delay between perturbed and unperturbed handstand would suggest an increase in sensory delay of $20 \pm 15 \mathrm{~ms}$ in unperturbed handstand. Collectively this would suggest a total of $43 \pm 20 \mathrm{~ms}$ to be subtracted from feedback time delay during unperturbed handstand to provide an estimate of neurological delay during that task. Of the two regression models used in this study the PS-PD model is superior as it provides better estimates of feedback time delay whilst accounting for the contribution of a passive stiffness mechanism. Further evidence of its superiority is provided when comparing the results of the PS-PD method between perturbed and unperturbed handstand, where a decreased contribution from the passive mechanism is found during unperturbed handstand.

The PS-PD model shows that the percentage of wrist torque arising from the passive stiffness mechanism drops from $26 \%$ in perturbed handstand to $5-6 \%$ in 
unperturbed handstand (Table 2). Previous research looking at total ankle torque during quiet stance suggests that an elastic torque caused by a passive stiffness mechanism at the ankle may account for up to $19 \%$ of total torque (Jacono et al., 2004). The Jacono et al. (2004) method of decomposing joint torque used ankle stiffness gains which where $0.7-0.9$ of critical (gravitational) torque; values taken from the literature (Casadio et al., 2003; Loram and Lakie, 2002). It has been suggested that ankle stiffness gains of 0.7-0.9 of critical torque are unrealistically high (van der Kooij et al., 2005), and gains of approximately 0.1 may be more appropriate (Peterka, 2002). The elastic torque estimated from Jacono et al. (2004) is sensitive to the ankle stiffness gain, and will scale linearly with the lower gain of 0.1 from $19 \%$ of total torque to approximately 2-3\%. Results from the present study suggest the contribution of passive stiffness during unperturbed handstands (5-6\%) is approximately twice that required during unperturbed standing. One possible cause for this may be the difference in functional range of motion at the ankle and wrist joints in the two tasks. In standing the ankle is positioned in a neutral position with a further $15^{\circ}$ of dorsiflexion and $40^{\circ}$ of plantar flexion available (Roaas and Andersson, 1982). In handstand the wrist joint is positioned towards the extreme of hyperextension, which is close to the limit of $78^{\circ} \pm 11^{\circ}$ extension that is available at the wrist (Gunal et al., 1996). The relationship between joint range of motion and passive force from the musculotendinous unit is exponential for both the ankle joint (Riener and Edrich, 1999) and the metacarpophalangeal joint (Esteki and Mansour, 1996). The hyperextended position of the wrist in handstands will result in the wrist and finger flexor muscles being stretched close to their limits, increasing the amount of force transmitted through the parallel elastic elements of the musculotendinous unit, explaining the increased contribution of passive stiffness during handstands.

Previous research has examined feedback time delay by applying perturbations to reveal the dynamics of the controller or plant within the system, then assuming these dynamics remain constant during unperturbed stance (van der Kooji et al., 2005). Perturbations degrade the predictability of the task and increase the necessity to respond rapidly and therefore stimulate shorter latency mechanisms (Loram et al., 2006). Results from the present study support this view, with a decrease in feedback time delay of approximately $20 \mathrm{~ms}$ (eyes open) and $32 \mathrm{~ms}$ (eyes closed) in perturbed compared to unperturbed handstands. The change is most likely due to a decrease in sensory delay caused by the increased motion imposed by the perturbation, with neurological and electromechanical delays expected to be approximately equal in each condition. High velocities of postural sway from platform translations will activate sensory receptors well above threshold levels, initiating fast stimulusresponse processes (Baratto et al., 2002). During the slower sway of unperturbed handstands sensory receptors may be stimulated below their physiological threshold, resulting in periods when motion goes undetected, leading to intermittent control (Bottaro et al., 2005, 2008).

Previous research has indicated typical EMG latencies are associated with specific voluntary or involuntary reflexes, which include: myotatic reflexes with a 45 50 ms latency (Nashner, 1976), tonic reflexes with a 50-70 ms latency (Wu and Zhao, 1997), and functional stretch reflexes with a 100-120 ms latency (Nashner, 1977). In addition, Nashner (1976) suggested that EMG latencies in the region of 180 to $200 \mathrm{~ms}$ are primarily due to visual or vestibular feedback. In the present study, EMG latencies of 137 to 192 ms for perturbed handstand were approximately 60-70 ms longer than those found in perturbed standing (Horak et al., 1989; Nashner et al., 1979). Although comparisons of EMG latencies in handstand to reflexes defined in standing should be made with caution, results indicate that handstand responses are 
due to a combination of functional stretch reflex of forearm musculature and feedback from visual or vestibular sensory systems. In addition, feedback time delays in unperturbed handstand showed there was a small, but statistically significant difference between eyes open and eyes closed conditions (Table 2). The small increase in feedback time delay of $13 \pm 13 \mathrm{~ms}$ from eyes open to eyes closed conditions indicates that while visual information is useful in maintaining handstand balance, it is not essential. It remains unclear if this small increase is due to an increase in neurological delay associated with increased reliance on vestibular inputs, or due to an increased sensory delay for other sensory organs to reach threshold levels.

\section{CONCLUSION}

Feedback time delay was evaluated in perturbed and unperturbed handstands. Delayed regression models provided feedback time delay estimates that were most in agreement with EMG latencies to a discrete platform perturbation. Cross correlations of wrist joint torque and COM displacement severely underestimated feedback time delays and are not recommended as an appropriate tool for determining feedback time delay in human balance. Feedback time delays were significantly lower during perturbed handstand compared to unperturbed handstand. This difference is expected to be due to a reduced sensory delay during perturbed handstands as a consequence of an increased COM velocity imposed from the external perturbation. The percentage of total wrist joint torque predicted from the PS-PD model coefficients highlighted some striking differences between perturbed and unperturbed handstand, supporting the view that balance measures in perturbed testing should not be assumed to be valid for unperturbed balance. The PS-PD delayed regression model is an appropriate tool for assessing feedback time delays and determining parameters that contribute to the production of wrist joint torque during perturbed and unperturbed handstand.

\section{REFERENCES}

Baratto L., Morasso P.G., Re C., \& Spada G. (2002). A new look at posturographic analysis in the clinical context: Sway-density versus other parameterization techniques. Motor Control, 6, 246-270.

Bottaro A., Casadio M., Morasso P.G., \& Sanguineti V. (2005). Body sway during quiet standing: Is it the residual chattering of an intermittent stabilization process? Hum Movement Sci, 24, 588-615.

Bottaro A., Yasutake Y., Nomura T., Casadio M., \& Morasso P. (2008). Bounded stability of the quiet standing posture: an intermittent control model. Hum Movement Sci, 27, 473-495.

Casadio M., Morasso P., \& Sanguineti V. (2003). Measurement of ankle stiffness. Gait Posture, 18, S7.

Cavanagh P.R. \& Komi P.V. (1979). Electromechanical delay in human skeletal muscle under concentric and eccentric contractions. Eur J Appl Physiol, 42, 159163.

Clark F.J., Burgess R.C., Chapin J.W., \& Lipscomb W.T. (1985). Role of intramuscular receptors in the awareness of limb position. J Neurophyisol, 54, 1529-1540. 
De Luca C.J., Gilmore L.D., Kuznetsov M., \& Roy S.H. (2010). Filtering surface EMG signal: Movement artefact and baseline noise contamination. J Biomech, 43, 15731579.

Dumas R., Aissaoui R., \& De Guise J.A. (2004). A 3D generic inverse dynamic method using wrench notation and quaternion algebra. Comput Methods Biomech Biomed Engin, 7(3), 159-166.

Esteki, A. \& Mansour, J.M. (1996). An experimentally based nonlinear viscoelastic model of joint passive moment. J Biomech, 29, 443-450.

Fitzpatrick R. \& McCloskey D.I. (1994). Proprioceptive, visual and vestibular thresholds for the perception of sway during standing in humans. J Physiol, 478.1, 173-186.

Gatev P., Thomas S., Kepple T., \& Hallett M. (1999). Feedforward ankle strategy of balance during quiet stance in adults. J Physiol, 514.3, 915-928.

Gunal, I., Kose, N., Erdogan, O., Gokturk, E., \& Seber, S. (1996). Normal range of motion of the joints of the upper extremity in male subjects, with special reference to side. The Journal of Bone and Joint Surgery, 78-A, 1401-1404.

Hodges P.W. \& Bui B.H. (1996). A comparison of computer-based methods for the determination of onset of muscle contraction using electromyography. Electroencephalogr Clin Neurophysiol, 101(6), 511-519.

Horak F.B., Diener H.C., \& Nashner L.M. (1989). Influence of central set on human postural responses. J Neurophysiol, 62, 841-853.

Horak, F.B. (2006). Postural orientation and equilibrium: What do we need to know about neural control of balance to prevent falls? Age and Ageing, 35, ii7-ii11.

Jacono M., Casadio M., Morasso P.G., \& Sanguineti V. (2004). The sway-density curve and the underlying postural stabilization process. Motor Control, 8, 292-311.

Kerwin, D.G. \& Trewartha, G. (2001). Strategies for maintaining a handstand in the anterior-posterior direction. Medicine and Science in Sports and Exercise, 33, 1182-1188.

Kiemel, T., Oie, K.S., \& Jeka, J.J. (2002). Multisensory fusion and the stochastic structure of postural sway. Biol Cybern, 87, 262-277.

Loram I.D. \& Lakie M. (2002). Direct measurement of human ankle stiffness during quiet standing: the intrinsic mechanical stiffness is insufficient for stability. J Physiol, 545, 1041-1053.

Loram I.D., Gawthrop P.J., \& Lakie M. (2006). The frequency of human, manual adjustments in balancing an inverted pendulum is constrained by intrinsic physiological factors. J Physiol, 577.1, 417-432.

Masani K., Popovic M.R., Nakazawa K., Kouzaki M., \& Nozaki D. (2003). Importance of body sway velocity information in controlling ankle extensor activities during quiet stance. J Neurophysiol, 90, 3774-3782.

Masani K., Vette A.H., \& Popovic M.R. (2006). Controlling balance during quiet standing: Proportional and derivative controller generates preceding motor commands to body sway position observed in experiments. Gait Posture, 23, 164172.

Morasso P. G. \& Schieppati, M. (1999). Can muscle stiffness alone stabilize upright standing? J Neurophysiol, 83, 1622-1626.

Morasso P.G., Baratto L., Capra R., \& Spada G. (1999). Internal models in the control of posture. Neural Networks, 12, 1173-1180. 
Moretti D.V., Babiloni F., Carducci F., Cincotti F., Remondini E., Rossini P.M., Salinari, S., \& Babiloni, C. (2003). Computerized processing of EEG-EOG-EMG artefacts for multi-centric studies in EEG oscillations and event-related potentials. Int J Psychophysiol, 47, 199-216.

Muraoka T., Muramatsu T., Fukunaga T., \& Kanehisa H. (2004). Influence of tendon slack length on electromechanical delay in the human medial gastrocnemius in vivo. J Appl Physiol, 96, 540-544.

Nashner L.M., Woollacott M., \& Tuma G. (1979). Organisation of rapid responses to postural and locomotor-like perturbations of standing man. Exp Brain Res, 36, 463-476.

Nashner, L.M. \& McCollum, G. (1985). The organization of human postural movements: A formal basis and experimental synthesis. The Behavioral and Brain Sciences, 8, 135-172.

Nashner, L.M. (1976). Adapting reflexes controlling the human posture. Exp Brain Res, 26, 59-72.

Nashner, L.M. (1977). Fixed patterns of rapid postural responses among leg muscles during stance. Exp Brain Res, 30, 13-24.

Peterka R.J. (2002). Sensorimotor integration in human postural control. J Neurophysiol, 88, 1097-1118.

Peterka, R.J. (2000). Postural control model interpretation of stabilogram diffusion analysis. Biol Cybern, 82, 335-343.

Preuss R. \& Fung J. (2004). A simple method to estimate force plate inertial components in a moving surface. J Biomech, 37, 1177-1180.

Pulkovski N., Schenk P., Maffiuletti N.A., \& Mannion A.F. (2008). Tissue Doppler imaging for detecting onset of muscle activity. Muscle Nerve, 37, 638-649.

Riener, R. \& Edrich, T. (1999). Identification of passive elastic joint moments in the lower extremities. J Biomech, 32, 539-544.

Roaas, A. \& Andersson, G.B.J. (1982). Normal range of motion of the hip, knee and ankle joints in male subjects, 30-40 years of age. Acta orthop. scand. 53, 205-208.

Runge, C.F., Shupert, C.L., Horak, F.B., \& Zajac, F.E. (1999). Ankle and hip postural strategies defined by joint torques. Gait Posture, 10, 161-170.

Slobounov, S.M. \& Newell, K.M. (1996). Postural dynamics in upright and inverted stances. J Applied Biomech, 12, 185-196.

Suzuki, Y., Nomura, T., Casadio, M., \& Morasso, P. (2012). Intermittent control with ankle, hip, and mixed strategies during quiet standing: A theoretical proposal based on a double inverted pendulum model. J Theor Biol, 310, 55-79.

Tillin N.A., Jimenez-Reyes P., Pain M.T.G., \& Folland J.P. (2010). Neuromuscular performance of explosive power in athletes versus untrained individuals. Med Sci Sport Exer, 42, 781-790.

Tillin N.A., Pain M.T.G., \& Folland J.P. (2013). Identification of contraction onset during explosive contractions. Response to Thompson et al. "Consistency of rapid muscle force characteristics: Influence of muscle contraction onset detection methodology" [J Electromyogr Kinesiol 2012; 22(6): 893-900]. J Electromyogr Kinesiol, 23(4), 991-994.

van der Kooij, H., van Asseldonk, E., \& van der Helm, F.C.T. (2005). Comparison of different methods to identify and quantify balance control. J Neurosci Meth, 145, 175-203. 
Vette, A.H., Masani, K., Nakazawa, K., \& Popovic, M.R. (2010). Neural-mechanical feedback control scheme generates physiological ankle torque fluctuation during quiet stance. IEEE Trans Neural Syst Rehabil Eng

Wade, M.G. \& Jones, G. (1997). The role of vision and spatial orientation in the maintenance of posture. Physical Therapy, 77, 619-628.

Winter D.A., Patla A.E., Ischac M.G., \& Gage W.H. (2003). Motor mechanisms of balance during quiet standing. J Electromyogr Kines, 13, 49-56.

Winter D.A., Patla A.E., Prince F., Ishac M., \& Gielo-Perczak K. (1998). Stiffness control of balance in quiet standing. J Neurophysiol, 80, 1211-1221.

Winter D.A., Prince F., Frank, J.S., Powell, C., \& Zabjek, K.F. (1996). Unified theory regarding $\mathrm{A} / \mathrm{P}$ and $\mathrm{M} / \mathrm{L}$ balance in quiet stance. J Neurophysiol, 75, 2334-2343.

Winter, D.A. (1995). Human balance and posture control during standing and walking. Gait Posture, 3, 193-214.

Wu G. and Zhao, W. (1997). The role of mechanoreceptive information in the stability of human upright posture: A theoretical consideration. Motor Control, 1, 3-19.

Yeadon M.R. \& Trewartha G. (2003). Control strategy for a hand balance. Motor Control, 7, 411-430.

Yeadon M.R. (1990). The simulation of aerial movement - II. A mathematical inertia model of the human body. J Biomech, 23, 67-74.

Zhou S., Lawson D.L., Morrison W.E., \& Fairweather I. (1995). Electromechanical delay in isometric muscle contractions evoked by voluntary, reflex and electrical stimulation. Eur J Appl Physiol, 70, 138-145. 